\title{
Gross Motor Function in Children with Congenital Zika Syndrome
}

\author{
Eliana Harumi Morioka Takahasi ${ }^{1,2}$ Maria Teresa Seabra Soares de Britto Alves ${ }^{2}$ \\ Marizélia Rodrigues Costa Ribeiro ${ }^{2}$ Valéria Ferreira Pereira Souza ${ }^{3}$ Vanda Maria Ferreira Simões ${ }^{2}$ \\ Marcella Costa Ribeiro Borges ${ }^{4}$ Gláucio Andrade Amaral ${ }^{1}$ Lillian Nunes Gomes ${ }^{5}$ Ricardo Khouri6,7 \\ Patricia da Silva Sousa ${ }^{3}$ Antônio Augusto Moura da Silva ${ }^{2}$
}

${ }^{1}$ Sarah Network of Neurorehabilitation Hospitals, São Luís, Maranhão, Brazil

2 Public Health Department, Federal University of Maranhão, São Luís, Maranhão, Brazil

${ }^{3}$ Reference Center on Neurodevelopment, Assistance and Rehabilitation of Children - NINAR, State Department of Health of the State of Maranhão, São Luís, Maranhão, Brazil

${ }^{4}$ Diagnostic Imaging Department, Federal University of São Paulo, São Paulo, São Paulo, Brazil

${ }^{5}$ Laboratory of Immunology Human, Department of Immunology, Institute of Biomedical Sciences, University of São Paulo, São Paulo, São Paulo, Brazil

${ }^{6}$ Laboratory of Vector-Borne Infectious Diseases, Gonçalo Moniz Institute, Salvador, Bahia, Brazil

${ }^{7}$ Department of Pathology and Legal Medicine, Faculty of Medicine, Federal University of Bahia, Salvador, Bahia, Brazil

Address for correspondence Eliana Harumi Morioka Takahasi, MD, Programa de Pós-Graduação em Saúde Coletiva, Rua Barão de Itapary, N 155, Centro, CEP: 65020-070, São Luís/MA, Brazil (e-mail: elimorioka@gmail.com).

Neuropediatrics 2021;52:34-43.

\begin{abstract}
Keywords

- Zika virus

- cerebral palsy

- nervous system

- malformations

Background Little information on gross motor function of congenital Zika syndrome (CZS) children is available.

Objectives To evaluate gross motor function in CZS children aged up to 3 years, and its associated factors and changes in a minimum interval of 6 months.

Methods One hundred children with CZS and cerebral palsy (36 with confirmed and 64 with presumed CZS) were evaluated with the Gross Motor Function Classification System (GMFCS) and Gross Motor Function Measure (GMFM-88/GMFM-66). Forty-six were reevaluated. Wilcoxon tests, Wilcoxon tests for paired samples, percentile scores, and score changes were performed.

Results Clinical and socioeconomic characteristics (except maternal age), GMFM scores and GMFCS classification of confirmed and probable cases, which were analyzed together, were similar. The mean age was 25.6 months ( \pm 5.5 ); the median GMFM- 88 score was 8.0 (5.4-10.8); and the median GMFM-66 score was 20.5 (14.8-23.1); 89\% were classified as GMFCS level V. Low economic class, microcephaly at birth, epilepsy, and brain parenchymal volume loss were associated with low GMFM-66 scores. The median GMFM-66 percentile score was 40 (20-55). On the second assessment, the GMFM-66 scores in two GMFCS level I children and one GMFCS level IV child improved significantly. In one GMFCS level III child, one GMFCS level IV child, and the group of GMFCS level $V$ children, no significant changes were observed.
\end{abstract}

received

May 4, 2020

accepted after revision

August 31, 2020

published online

October 27, 2020 (c) 2020. Thieme. All rights reserved.

Georg Thieme Verlag KG,

Rüdigerstraße 14,

70469 Stuttgart, Germany
DOI https://doi.org/

10.1055/s-0040-1718919. ISSN 0174-304X. 
Conclusions Almost all CZS children had severe cerebral palsy; in the third year of life, most presented no improvement in gross motor function and were likely approaching their maximal gross motor function potential.

\section{Introduction}

Until 2015, Zika virus (ZIKV) infection reports in humans were isolated and sporadic, and ZIKV infection was mainly

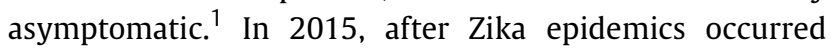
throughout the Americas, intrauterine ZIKV infection was found to be a cause of microcephaly and serious brain anomalies. $^{2,3}$

A distinct pattern of birth defects, called congenital Zika syndrome (CZS), was described. ${ }^{4} \mathrm{CZS}$ has been observed in approximately $10 \%$ of cases of ZIKV infection in pregnancy, with presentation ranging from mild symptoms to microcephaly with multiple organ involvement; its full spectrum is still unknown. ${ }^{5}$

Defining cases is challenging in ZIKV studies. Molecular tests used to detect viral genomic material are the preferred method of diagnosis because they can provide confirmed evidence of infection, according to the Centers for Disease Control and Prevention (CDC), but due to the temporal nature of ZIKV RNA in serum and urine, these tests often produce false-negative results. ${ }^{6}$ ZIKV immunoglobulin (Ig) M antibody testing, followed by the plate reduction neutralization test (PRNT) for ZIKV, expands the diagnostic window and is recommended by the CDC in certain situations. However, it has recently been shown that negative PRNT results do not exclude the diagnosis of CZS, since among mothers who were ZIKV-positive according to qRT-PCR (real-time quantitative polymerase chain reaction), $51.5 \%$ had a negative PRNT result. $^{7}$ Therefore, laboratory testing has been integrated with clinical knowledge of CZS and presumed CZS is commonly diagnosed. ${ }^{8-11}$

The motor function of children with probable CZS and cerebral palsy (CP) can be very compromised when compared with populations with typical development according to normative tests. ${ }^{12-14} \mathrm{CZS}$ children often present with $\mathrm{CP}^{15,16}$ that differs from other congenital infections because of the following features: (1) severe microcephaly with partially collapsed skull; (2) thin cerebral cortices with subcortical calcifications; (3) macular scarring and focal pigmentary retinal mottling; (4) congenital contractures; and (5) marked early hypertonia and symptoms of extrapyramidal involvement, ${ }^{17}$

Criteria-referenced tests specifically developed for CP, such as the Gross Motor Function Measure (GMFM-88 and GMFM-66), ${ }^{18}$ are highly appropriate to describe motor function and detect changes over time. Recent studies on gross motor function in CZS children administered the GMFM-88 in small samples and presented transversal data. ${ }^{9,19,20}$ The associations of severe cortical malformation and small head circumference at birth, ${ }^{9,20}$ early maternal infection, ${ }^{20}$ and epilepsy and dysphagia ${ }^{19}$ with decreased motor function were described.
One longitudinal study revealed evidence of marginal improvement in motor function in the first 2 years of life in children with probable CZS. ${ }^{11}$ Additional studies with older children using appropriate instruments and larger samples are required to better understand motor abilities, limitations, and factors associated with a poor prognosis in CZS children. ${ }^{9,11,19}$

The objective of this study was to evaluate gross motor function and its associated factors in a larger sample of children with CZS aged up to 3 years using the GMFM and to determine changes in this domain with a minimum interval of 6 months.

\section{Methods}

A prospective cohort was conducted between September 2017 and February 2019 at the Reference Center on Neurodevelopment, Assistance and Rehabilitation of Children-NINAR, affiliated with the State Department of Health of the State of Maranhão.

The study was part of the project "Congenital Zika Syndrome, Seroprevalence, and Spatial and Temporal Analysis of Zika and Chikungunya Virus in Maranhão," approved by the Research Ethics Committee of the University Hospital of the Federal University of Maranhão (approval number 2.111.125).

\section{Participants}

The inclusion criteria consisted of children who attended follow-ups with the multidisciplinary team at NINAR and who had received a diagnosis of CZS confirmed by a PRNT for ZIKV with a cutoff value of 90\% (PRNT90; 40 children) or a presumed diagnosis (70 children) based on computed tomography (CT) findings, ZIKV IgM detection, and serology tests for other congenital infections.

PRNT90 was conducted in the Laboratory of Vector-Borne Infectious Diseases (LEITV)-Gonçalo Muniz Institute (IGM)/ Oswaldo Cruz Foundation (FIOCRUZ)/Bahia. This test was performed based on a previously reported protocol, ${ }^{21}$ with minor modifications. PRNT90 was performed to determine the maximum serum dilution (1:8 to $1: 4096)$ needed to reduce ZIKV plaque formation by $90 \%$ in Vero cells. For this, the ZIKV PE/243 virus strain that was isolated in Brazil was used. All sera were heat-inactivated $\left(56^{\circ} \mathrm{C}, 30\right.$ minutes) prior to neutralization testing. The serum samples were diluted on a plate with modified Dulbecco Eagle medium containing 2\% fetal calf serum and $1 \%$ of penicillin/streptomycin. Next, $250 \mu \mathrm{L}$ of virus $(100 \mathrm{ffu} / \mu \mathrm{L})$ was added to each well containing diluted serum (1:1). The serum and virus dilutions were then incubated at $37^{\circ} \mathrm{C}$ for 60 minutes. A final volume of $200 \mu \mathrm{L}$ of each serum and virus dilution was transferred to a 
well containing Vero cells and then incubated at $37^{\circ} \mathrm{C}$ for 60 minutes. Following incubation, $300 \mu \mathrm{L}$ of $0.3 \%$ agarose solution was added and plates were reincubated at $37^{\circ} \mathrm{C}$ for 5 days. Reactions were then revealed using a $2 \%$ naphthol blue-black solution. Titers $\geq 10$ were considered positive.

Brain CT scans were evaluated by two experienced radiologists to identify specific features associated with CZS, ${ }^{22,23}$ including brain parenchymal volume loss, calcifications in the gray-white matter junction, ventricular enlargement, delayed cortical development, cerebellar and/or brainstem malformations, and a hypoplastic or absent corpus callosum. Children with radiological findings compatible with CZS in addition to positive IgM for ZIKV (three children), negative serology ( 33 children) or inconclusive serology (34 children) for other congenital infections (syphilis, toxoplasmosis, rubella, cytomegalovirus, and herpes simplex virus) were presumed to have CZS. Children for whom neuroimaging examinations showed no abnormalities or signs of other causes for $\mathrm{CP}$, who had no neuroimaging examination results available, or who had positive serological detection of other congenital infections were not included.

Two children died before data collection, two were excluded from this study due to concomitant diagnosis of conditions that interfere with motor performance (hydrocephalus and Dandy-Walker syndrome), two were clinically ill, and four were unavailable to complete the motor function assessment. Therefore, for the purposes of this study, 100 children with $\mathrm{CZS}$ and $\mathrm{CP}$ were evaluated, including 36 with confirmed and 64 with presumed CZS.

The mothers or guardians of the children who met the inclusion criteria were invited to participate in the study and were included after signing an informed consent form.

\section{GMFCS, GMFM-88, and GMFM-66}

The children's spontaneous movements were evaluated by three trained physical therapists and an occupational therapist who applied the GMFCS ${ }^{24}$ version validated in Portuguese $^{25}$ and the GMFM-66 and GMFM-88. ${ }^{18}$ The evaluations were video-recorded and later scored by the same experienced physiotherapist (E.H.M.T.) who was trained in GMFM66 and GMFM-88 scoring. The GMFCS is a five-level patternrecognition system to describe and classify the severity of movement disabilities in children with CP. Level I represents the best gross motor abilities (CP children and youth who walk without limitations), and level $\mathrm{V}$ represents the poorest function (children who require a wheelchair). ${ }^{24}$ The GMFM88 is an ordinal scale that consists of five dimensions: (A) lying and rolling, (B) sitting, (C) crawling and kneeling, (D) standing, and (5) walking, running, and jumping. All items were classified on a 4-point scale and the raw scores were converted into percentages. The GMFM- 88 provides a more detailed description of motor function in young children or highly impaired children. The GMFM-66, on the other hand, is an interval measurement tool developed using Rasch analysis of the GMFM- $88,{ }^{26}$ thereby making comparisons of changes in subjects, as well as changes over time in subjects, more reliable and accurate. The Gross Motor Ability Estimator (GMAE-2) was used to calculate the GMFM-66 scores, which were also analyzed as percentiles according to the child's GMFCS classification. ${ }^{27}$ A convenience sample of 46 children repeated the GMFM-66 assessment after a minimum interval of 6 months.

\section{Clinical and Socioeconomic Characteristics}

A standardized questionnaire was completed by mothers or guardians, providing socioeconomic data (mother's education level and age at the beginning of gestation, place of family residence at the time of pregnancy, monthly family income, and economic classification according to the Criterion of Economic Classification Brasil $\left(\mathrm{CCEB}^{28}\right)$; presence of symptoms compatible with ZIKV infection during the gestational period, type of delivery, gestational age at birth (in weeks); and the child's head circumference, length, and weight at birth (the first two in centimeters and the last in grams). The CCEB is based on the accumulation of material goods and education level of the household head and groups of people into classes ( $A, B, C, D$, or $E$ ) according to the scores obtained. Class " $A$ " refers to the highest socioeconomic status and class " $E$ " refers to the lowest. The head circumference was classified in z-scores according to the INTERGROWTH-21st tables ${ }^{29}$ to determine the presence of macrocephaly (z-score $>2$ ), normocephaly $(2 \leq \mathrm{z}$-score $\leq-2)$, or microcephaly $(z$-score $<-2)$. The presence and degree of brain parenchymal volume loss (mild to moderate or severe) were determined in head CT. All children were diagnosed with $\mathrm{CP}$ and classified according to topography and the presence of pyramidal (hypertonia, clonus, hyperreflexia, and increased archaic reflexes) or extrapyramidal signs (tonus fluctuation and asymmetric dyskinesias in the extremities that were absent during sleep) $)^{30}$ by the chief child neurologist of NINAR.

Data on the presence of symptomatic epilepsy were collected from medical records.

\section{Statistical Analysis}

The distribution of categorical variables and the medians and interquartile ranges (IQRs) of numerical variables were analyzed.

Chi-square tests and Wilcoxon tests were performed to compare children with confirmed and presumed CZS diagnoses.

Wilcoxon tests were used to evaluate associations between GMFM-66 baseline scores and the independent variables, which were transformed into dichotomous variables. The GMFM-66 scores of children aged 24 months or older were analyzed as percentiles using motor development curves as references. ${ }^{27}$

The Wilcoxon test for paired samples was used to compare repeat GMFM-66 scores with baseline scores. Change scores by age and GMFCS levels were calculated.

For all tests, a $5 \%$ level of significance was adopted. The statistical analysis was conducted in Stata, version 14.0 (Stata Corp., College Station, Texas, United States).

\section{Results}

Maternal age at the beginning of gestation was the only characteristic with significant difference $(p=0.044)$ between confirmed (median maternal age of 21.5 years; IQR: $19-26.5$ ) 
and presumed CZS children (median: 26 years; IQR 20-31); the other clinical and demographic characteristics were similar (-Supplementary Table S1, available online only).

Most children were male (59.0\%), were delivered by cesarean (52\%) and were microcephalic (56.0\%), presented tetraparetic CP (88.0\%), exhibited both pyramidal and extrapyramidal signs (67.0\%), had symptomatic epilepsy (89.0\%), and showed brain parenchymal volume loss (68.0\%). Prematurity and low birth weight were not uncommon (13.0 and $22.0 \%$, respectively). At the time of pregnancy, $76.0 \%$ of the families were living in urban areas. Sixty-three percent of mothers had completed high school or incomplete higher education and $52.0 \%$ had experienced symptoms of viral infection mainly in their first trimester of pregnancy (52. \%). Low socioeconomic status (economic classes D-E) was the most common status (46.0\%). Families of confirmed and presumed CZS children had similar monthly income (median: US\$250; IQR 200-500; median: US\$300, IQR 232.50-500, respectively; $p=0,739$ ).

At the first gross motor function evaluation, the mean age was similar $(p=0.974)$ among confirmed CZS children (25.6 \pm 5.1 months) and presumed CZS children $(25.5 \pm 5.8$ months). Most children were classified as GMFCS level V (89.0\%; - Table 1 ). The proportion of mild/moderate cases (GMFCS levels I-III) and severe cases (GMFCS IV-V) were similar between confirmed and presumed CZS children $(p=0.444)$. The baseline GMFM-88 and GMFM-66 scores of confirmed (median GMFM-88 score: 7.9; median GMFM66 score: 19.3 ) and presumed CZS children (median GMFM88 score: 8.0; median GMFM-66 score: 20.5$)$ showed no significant difference $(p=0.747$ and $p=0.635$ respectively; - Table $\mathbf{1}$ and - Fig. $\mathbf{1}$ ).

Considering that nearly all the clinical and socioeconomic characteristics, GMFM scores, and GMFCS classifications (-Table 2) from confirmed and probable cases were similar, the groups were combined in one group for subsequent analyses.

There were no differences in the GMFM-66 scores according to place of family residence during pregnancy, presence of infectious disease symptoms during pregnancy, type of delivery, gestational age, birth weight, and presence of pyramidal signs versus combined pyramidal and extrapyramidal signs (-Supplementary Table S2, available online only).

Children born with microcephaly had significantly lower GMFM-66 scores $(p=0.014)$ than children born with normocephaly. Lower scores on the GMFM-66 were also observed in children who belonged to lower economic classes ( $p=0.007$ ), who presented symptomatic epilepsy $(p<0.001)$, and who had brain parenchymal volume loss $(p<0.001)$ (-Supplementary Table S2, available online only).

All children with microcephaly at birth were classified as GMFCS level V. The other features of poor prognosis associated with low GMFM-66 scores, including lower economic class, symptomatic epilepsy, and brain parenchymal volume loss, were observed in children classified as GMFCS levels I-V (-Fig. 2).

All 46 reevaluated children received the same GMFCS rating at the second assessment. The median interval between evaluations was 8 months (6-14 months). In the second evaluation, the average age of children was 31.4 months of age (17-38 months). Most children were between 25 and 48 months old and classified as GMFCS level V (-Table 2).

Two (4.3\%) children were classified as GMFCS level I and presented changes in GMFM-66 scores greater than the measurement error, with change scores of 9.8 and 11.5 . Repeated motor assessments for one (2.2\%) GMFCS level III (change score of 0.4 ) child and one (change score of -0.6) out of two GMFCS level IV children (4.3\%) were similar (overlapping 95\% confidence intervals [CIs]). The other child classified as GMFCS level IV had a significant increase in the GMFM-66 score, with a change score of 4.5. In the group of GMFCS level V children (89.2\%), no significant differences were observed in the GMFM-66 scores of children who were initially evaluated at less than 2 years old (41.3\%; median change score of 0.6 ; IQR: $0-2.5 ; p=0.050$ ) or between 2 and 4 years old (47.9\%; median change score of 1.5 ; IQR: $0-2.2$; $p=0.060$ ) (-Table 3).

The median GMFM-66 score percentile ${ }^{27}$ was 40 (IQR: 20-55) among the 109 evaluations performed when children were 24 months old or older. One GMFCS level I child scored

Table 1 Comparison of Gross Motor Function Measure (GMFM) scores and Gross Motor Function Classification System (GMFCS) frequencies between confirmed and presumed congenital Zika syndrome (CZS) children (Sao Luis, Maranhao, 2017-2019)

\begin{tabular}{|l|l|l|l|l|}
\hline & Total $(\boldsymbol{n}=\mathbf{1 0 0})$ & CZS confirmed by PRNT90 $(\boldsymbol{n}=\mathbf{3 6})$ & Presumed CZS $(\boldsymbol{n}=\mathbf{6 4})$ & $p$-Value \\
\hline GMFM scores median (IQR) & $8.0(5.4-10.8)$ & $7.9(5.8-9.9)$ & $8.0(4.9-11.9)$ & 0.747 \\
\hline GMFM-88 & $20.5(14.8-23.1)$ & $19.3(15.4-21.6)$ & $20.5(14.8-24.0)$ & 0.635 \\
\hline GMFM-66 & \multicolumn{5}{|l|}{} \\
\hline GMFCS level & $1(2.8 \%)$ & $2(3,1 \%)$ & 0.309 \\
\hline GMFCS level I & $3(3.0 \%)$ & - & - & \\
\hline GMFCS level II & - & 0 & $2(3.1 \%)$ & \\
\hline GMFCS level III & $2(2.0 \%)$ & $1(2.8 \%)$ & $5(7.8 \%)$ & \\
\hline GMFCS level IV & $6(6.0 \%)$ & $34(94.4 \%)$ & $55(86.0 \%)$ & \\
\hline GMFCS level V & $89(89.0 \%)$ &
\end{tabular}

Abbreviations: CZS, congenital Zika syndrome; IQR, interquartile range; PRNT90, plaque reduction neutralization test with cutoff value of $90 \%$ (PRNT90). 

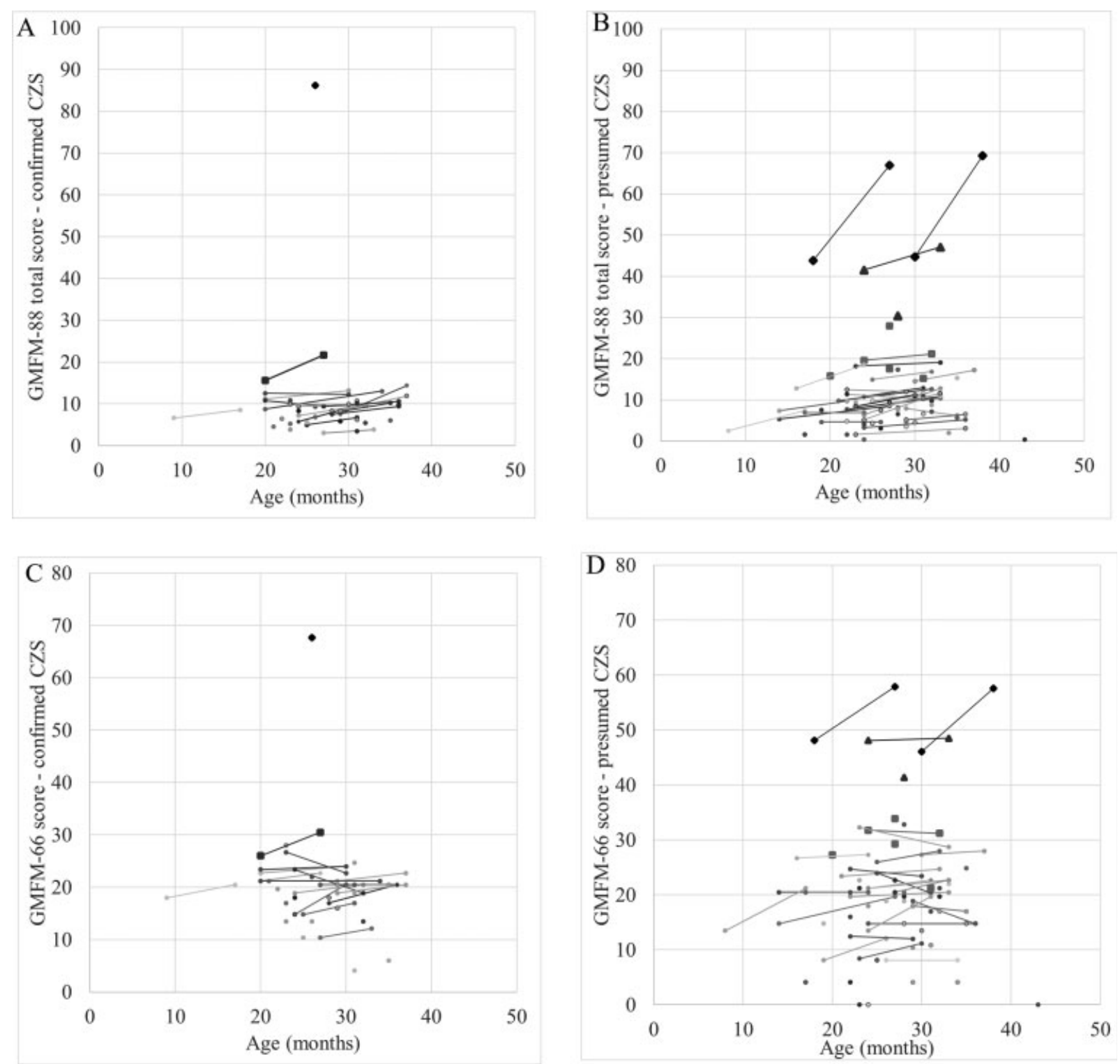

Fig. 1 (A) Gross Motor Function Measure-88 (GMFM-88) total scores of children with confirmed congenital Zika syndrome (CZS); (B) GMFM-88 total scores of children with presumed CZS; (C) GMFM-66 scores of children with confirmed CZS; (D) GMFM-66 score of children with presumed CZS. Gross Motor Classification System (GMFCS) level I; $\Delta$ GMFCS level III; $\mathbf{\square}$ GMFCS level IV; • GMFCS level V. São Luis, Maranhão, $2017-2019$.

in the 3rd and 20th percentiles, respectively; one in the 35th and the last in the 85th percentile. One GMFCS level III child scored in the 70th and 60th percentiles, respectively, and the other scored in 15th percentile. Among five children with GMFCS level IV, scores ranged from the 3rd to 50th percentiles. Among GMFCS level V children, $71.9 \%$ were at or below the 50th percentile.

\section{Discussion}

In the present study, motor function in children with CZS was described, including classification of the severity of $\mathrm{CP}$ and factors associated with poor motor function and gross motor trajectories until the third year of life. The most common phenotype observed was severe tetraparetic $\mathrm{CP}$, with $95 \%$ of children classified as GMFCS level V or IV. The proportion of
GMFCS level V children (89\%) was higher than those reported by Carvalho et $\mathrm{al}^{8}(40.2 \%)$ and Frota et $\mathrm{al}^{19}(71.7 \%)$, but similar to those in the study of Melo et $\mathrm{al}^{9}(81 \%)$ and Ventura et $\mathrm{al}^{11}$ (96.1\%). The differences are possibly explained by distinct inclusion criteria and smaller and younger average ages in previous studies. In the present sample, $67 \%$ of children were more than 24 months old, when the GMFCS classification is more precise. ${ }^{25}$ As expected, the majority of GMFCS level IV and V children could not assume crawling or standing positions, with poor prognosis for walking. ${ }^{24}$

Nevertheless, three children presented mild symptoms of CP and were classified as GMFCS level I; those children were able to walk.

The baseline GMFM-88 score was low (median of 8.0). However, Frota et $\mathrm{al}^{19}$ and Melo et $\mathrm{al}^{9}$ reported even lower GMFM-88 scores (median score of 4.9 in 46 24-month-old 
Table 2 Baseline Gross Motor Function Measurement (GMFM88 and GMFM-66) total scores and scores per GMFM-88 dimension in children with confirmed and presumed congenital Zika syndrome (CZS) according to the classification level of the Gross Motor Function Classification System (GMFCS).

\begin{tabular}{|c|c|c|}
\hline GMFM assessment & $\begin{array}{l}\text { Minimum; } \\
\text { maximum }\end{array}$ & Median (IQR) \\
\hline \multicolumn{3}{|l|}{ GMFM-88 } \\
\hline Level I $(n=3)$ & $43.8 ; 86.2$ & $44.7(43.8-86.2)$ \\
\hline Level II $(n=0)$ & - & - \\
\hline Level III $(n=2)$ & $30.3 ; 41.5$ & $35.9(30.3-41.5)$ \\
\hline Level IV $(n=6)$ & $15.2 ; 28.0$ & $18.6(15.6-19.6)$ \\
\hline Level V $(n=89)$ & $0.4 ; 18.2$ & $7.5(5.2-9.5)$ \\
\hline \multicolumn{3}{|l|}{ Dimensions } \\
\hline (A) Lying and rolling & $2 ; 100.0$ & $27.5(19.6-37.3)$ \\
\hline (B) Seating & $0 ; 90.0$ & $11.7(6.7-16.7)$ \\
\hline $\begin{array}{l}\text { (C) Crawling and } \\
\text { kneeling }\end{array}$ & $0 ; 71.4$ & $0(0-0)$ \\
\hline (D) Standing & $0 ; 79.5$ & $0(0-0)$ \\
\hline $\begin{array}{l}\text { (E) Walking, running, } \\
\text { and jumping }\end{array}$ & $0 ; 90.3$ & $0(0-0)$ \\
\hline \multicolumn{3}{|l|}{ GMFM-66 } \\
\hline Level I $(n=3)$ & $46.1 ; 67.7$ & $48.1(46.1-67.7)$ \\
\hline Level II $(n=0)$ & - & - \\
\hline Level III $(n=2)$ & $41.4 ; 48.1$ & $44.8(41.4-48.1)$ \\
\hline Level IV $(n=6)$ & $21.2 ; 33.9$ & $28.3(26.0-31.8)$ \\
\hline Level V $(n=89)$ & $0 ; 32.8$ & $18.9(14.8-21.2)$ \\
\hline
\end{tabular}

Abbreviation: IQR, interquartile range.

Note: The mean age of the children was 25.6 months, ranging from 8 to 43 months ( $n=100$ ). São Luís, Maranhão, 2017-2019.

children and 6.5 in 59 children with a maximum age of 13.2 months, respectively). GMFM-66 scores were not calculated in those studies,

An analysis of percentiles of scores from the GMFM-66, which has not been applied in previous studies, ${ }^{10,15}$ showed that most CZS children presented poor gross motor function (median GMFM-66 percentile score of 40) when compared with the median performance of other $\mathrm{CP}$ children with the same age and GMFCS classification.

In the reassessment of 46 of the 100 CZS children, limited gross motor gains at median age of 31.4 months was observed when compared with $\mathrm{CP}$ children. Longitudinal data on motor function of CZS are scarce; Ventura et al ${ }^{11}$ described presumed CZS children up to 24 months old. GMFCS level I children showed significant improvements in motor function, but their performance was no better than that observed in the typical course of static encephalopathy. One child had a change score (9.8) according to expected $(11.6 \pm 3.2)^{27}$ at the age of 2 years and 3 months, but the GMFM-66 score was in the 35th. The other child with GMFCS level I had a change score that exceeded the expected gains
(11.5; reference value: $4.5 \pm 3.6){ }^{27}$ Nonetheless, their GMFM-66 score at 3 years and 2 months old kept them in a low percentile ranking: it changed from the 3rd to the 20th percentile.

The GMFM-66 score of the GMFCS level III child dropped from the 70th to near the 60th percentile by the age of 2 years and 9 months, with no significant score change between the two evaluations possibly indicating a deceleration in motor development not expected at this age in children in this classification. GMFCS level III children are expected to continue showing improvement in their gross motor function and reach $90 \%$ of their potential until the age of 3.7 years. ${ }^{31}$

GMFCS level IV children showed substandard motor function when compared with CP curves. One GMFCS level IV child had a change score of 4.5 , but the GMFM- 66 score was only in the 30th percentile at 2 years and 3 months old. The other GMFCS level IV child had a small decrease in their GMFM-66 score, in the 30th percentile by the age of 2 years and 8 months.

For CZS children classified as GMFCS level V, GMFM-66 scores were statistically similar between the subsequent evaluations, with median GMFM-66 scores at the second assessment of 21.3 for the younger and 19.8 for the older group. The younger group was initially evaluated at a median age of 20 months and then at a median age of 30 months; the older group underwent the first assessment at a median age of 26.5 months, and the second assessment was performed at a median age of 33 months. CP children with the same GMFCS classification present a GMFM-66 limit score of 22.3 (95\% CI: 20.7-24.0) and usually reach their GMFM-66 score limit at an older age (32.4 months; 95\% CI: 24-44.4 months). ${ }^{31}$ These findings suggest that CZS children classified as GMFCS level $\mathrm{V}$ tend to reach their maximal gross motor function potential relatively early, by their second birthday, and they tend to underperform when compared with CP children with the same GMFCS classification.

The present study makes important contributions to understanding factors affecting motor function in CZS children, a field with incipient knowledge. ${ }^{8,9}$ Epilepsy was associated with decreased motor function, probably reflecting greater central nervous system involvement. Symptomatic epilepsy was present in the majority of the sample; this result supports the previously described results in children with probable CZS. ${ }^{12,16}$

Brain parenchymal volume loss was observed in $82.9 \%$ of those with available cerebral CT results and was associated with low GMFM-66 scores. This radiological sign can be identified early in prenatal ultrasound or postnatal exams ${ }^{32}$ and can be another useful tool to help clinicians to identify poor motor prognosis.

Normal head circumference at birth did not exclude the presence of motor impairment, but children with microcephaly had worse gross motor function than those born with normocephaly, with a statistically significant difference in GMFM-66 scores. Similar findings were reported by other authors. ${ }^{8,9}$ Among children with a known head circumference at birth, $66.3 \%$ had microcephaly at birth, a proportion within the range reported by previous studies (39.7-88.6\%). ${ }^{4,10,33}$ 

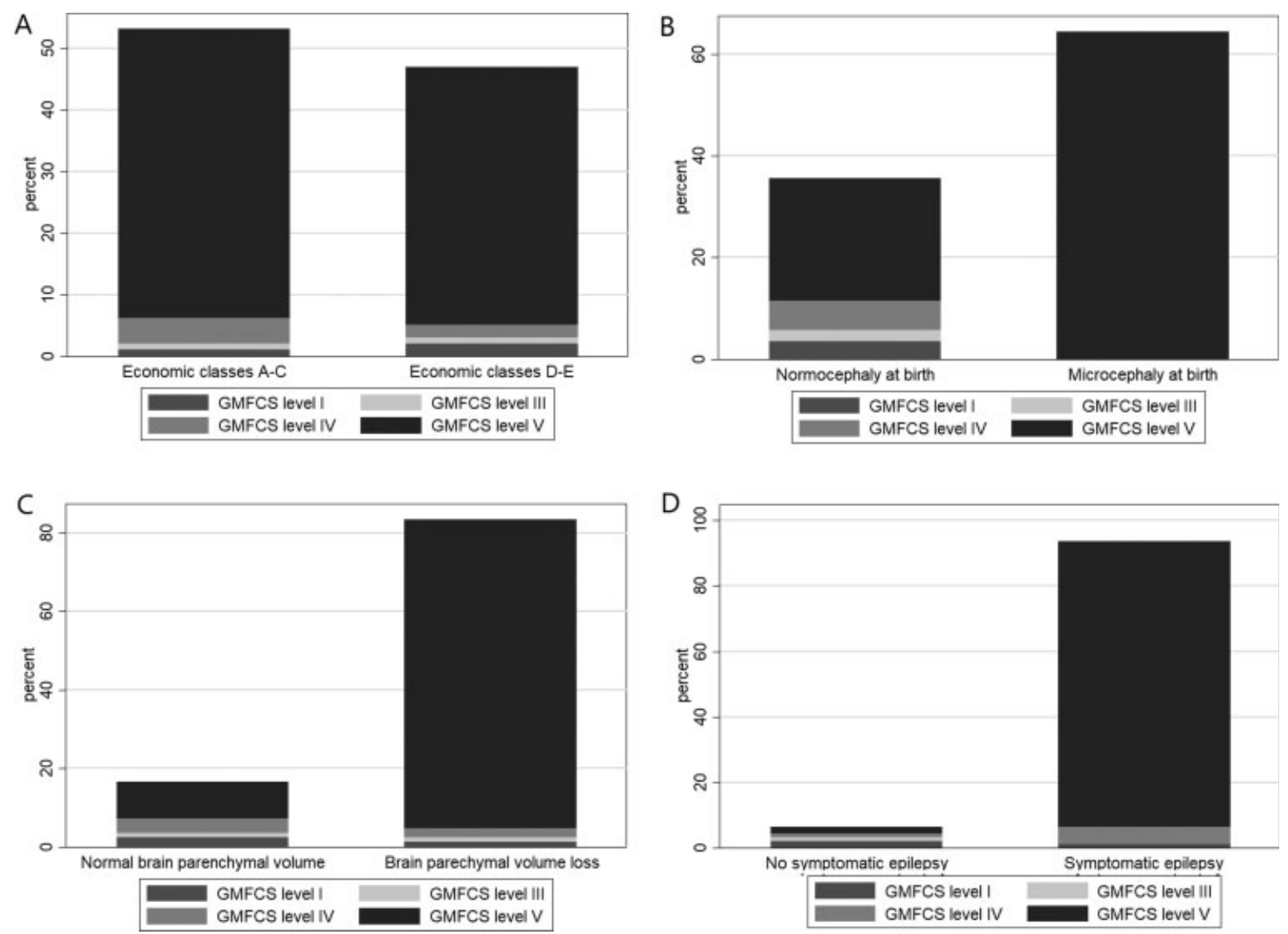

Fig. 2 Gross Motor Function Classification System (GMFCS) levels according feature associated with a poor prognosis. São Luís, Maranhão, 2017-2019. Economic classification according to the Criterion of Economic Classification Brasil-the letter "A" refers to the highest economic status and the letter " $E$ " refers to the lowest.

Table 3 Gross Motor Function Measure (GMFM-66) scores in children with two assessments, according to the Gross Motor Function Classification System (GMFCS) classification and age category in the first assessment (São Luís, Maranhão, 2017-2019)

\begin{tabular}{|c|c|c|}
\hline GMFCS classification & 1st assessment & 2nd assessment \\
\hline \multicolumn{3}{|l|}{ GMFCS level I $(n=2)$} \\
\hline $\begin{array}{l}\text { Child A (under } \\
2 \text { years old) }\end{array}$ & $48.1(45.9-50.3)$ & $57.9^{\mathrm{b}}(55.6-60.2)$ \\
\hline Child B (2-4 years old $)^{a}$ & $46.1(44.0-48.1)$ & $57.6^{\mathrm{b}}(55.3-59.9)$ \\
\hline \multicolumn{3}{|l|}{ GMFCS level III $(n=1)$} \\
\hline Child C ( $2-4$ years old $)^{a}$ & $48.1(45.9-50.3)$ & $48.5(46.3-50.7)$ \\
\hline \multicolumn{3}{|l|}{ GMFCS level IV $(n=2)$} \\
\hline $\begin{array}{l}\text { Child D (under } \\
2 \text { years old) }\end{array}$ & $26.0(22.1-29.1)$ & $30.5^{\mathrm{b}}(26.7-34.3)$ \\
\hline Child E (2-4 years old) ${ }^{\mathrm{a}}$ & $31.8(28.1-35.5)$ & $31.2(27.5-34.9)$ \\
\hline \multicolumn{3}{|l|}{ GMFCS level V $(n=41)$} \\
\hline $\begin{array}{l}\text { Under } 2 \text { years } \\
\text { old }(n=19)^{c}\end{array}$ & $21.2(18.0-23.4)$ & $21.2(20.5-24.0)$ \\
\hline $2-4$ years old $(n=22)^{c}$ & $19.7(14.8-21.2)$ & $20.5(17.0-22.7)$ \\
\hline
\end{tabular}

${ }^{\mathrm{a}} \mathrm{GMFM}-66$ score (95\% confidence interval).

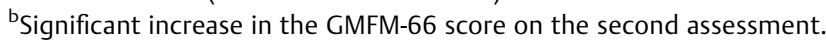
${ }^{\mathrm{c}}$ Median GMFM-66 score (interquartile range).
An unprecedented association between lower economic level and severe motor function was observed. Recent studies have shown that low yellow fever vaccination coverage ${ }^{34}$ and previous infections by viruses or other etiologic agents, ${ }^{35}$ factors to which lower socioeconomic classes are more exposed, can increase the severity of neurological involvement in congenital ZIKV infection. Moreover, CZS results in high-magnitude disabilities or difficulties in function and body structure, activity, and participation in daily life activities, ${ }^{36}$ creating several demands that are potentially difficult to meet in families with low socioeconomic status. The Brazilian National Health System offers basic care and specialized rehabilitation treatment ${ }^{37}$; however, this system needs to be continually improved to minimize the impact of CZS on the health and quality of life of children and their families.

Using the PRNT90 test for ZIKV was a strength of the study; this test provides robust evidence for ZIKV infection. ${ }^{7}$ However, it has recently been shown that negative PRNT results do not exclude the diagnosis of CZS. ${ }^{7}$ Therefore, no known specific criteria are capable of identifying all cases of CZS; a combination of clinical and risk assessments, clinical knowledge, and laboratory testing to create hierarchical classes of evidence of ZIKV infection, ${ }^{7}$ as adopted in this study, is necessary. ${ }^{6}$ Almost all clinical and socioeconomic characteristics (except for maternal age at the beginning of 
gestation), GMFM scores, and GMFCS classifications from confirmed and presumed CZS were similar, suggesting that CZS manifests similarly in children with different levels of ZIKV infection evidence, supporting the decision of combining both groups for the analysis.

Another strength of the study is the fact that gross motor function was evaluated with the gold standard tools for CP evaluation, the GMFM-88 and GMFM-66, which are widely used in the literature due to their high validity, reliability, and sensitivity to changes, ${ }^{26}$ in a larger sample than those evaluated in previous studies. ${ }^{9,11,19,20}$ The 88 version has additional items and allows separate evaluation of the different dimensions, providing a detailed picture for younger and severely impaired children. ${ }^{26}$ The GMFM-66, on the other hand, enables better comparison over time, among groups and with published for normative of $\mathrm{CP}$ children. ${ }^{27}$ The use of both the GMFM versions resulted in a broader and more reliable assessment of children's motor function in CZS than using either version alone. Data on follow-up on gross motor function in CZS children aged up to 3 years, as presented in this study, are scarce; the few studies available are generally restricted to the second year of life. ${ }^{11,26}$ Children assessed in this study can be reevaluated in future studies, expanding the knowledge about motor function in children with CZS.

There are some limitations to consider in this study. First, some factors that could affect motor function, such as visual and auditory impairment, were not analyzed. Second, families with highly impaired children are more likely to seek medical care at the rehabilitation center than those with mildly impaired children; thus families with children with mild presentations may be underrepresented in this study. Third, the number of children classified as GMFCS I-III was not large enough to allow further statistical analysis of the prognostic factors and gross motor function curve. Therefore, the sample size of this study ( 100 children) and the recruitment from a single rehabilitation center (convenience sample) preclude the generalization of the results, but in a scenario of limited knowledge about motor function in CZS children, this study provides a robust contribution to elucidating the impact of this congenital infection on the nervous system of developing fetuses.

\section{Conclusion}

Almost all children with CZS had severe CP and were considered as having with GMFCS level IV or V, with limited motor function. Gross motor function was poorer than the median performance of other $\mathrm{CP}$ children with the same age and GMFCS classification. A lower economic class, microcephaly at birth, symptomatic epilepsy, and brain parenchymal volume loss were associated with decreased gross motor function. In the third year of life, most children with severe $\mathrm{CP}$ presented no improvement in gross motor function and were probably approaching their maximal gross motor function potential.

Table of Contents Summary

Gross motor function and its associated factors of 100 children with confirmed or presumed CZS were evaluated.
Forty-six children were reevaluated in a minimum interval of 6 months.

\section{Author Contribution}

Ms. Eliana Harumi Morioka Takahasi conceived and designed the study, collected the data on motor function, performed the analysis, drafted the initial manuscript. Professor Maria Teresa Seabra Soares de Britto e Alves, Professor Marizélia Rodrigues Costa Ribeiro and Professor Vanda Maria Ferreira Simões conceived and designed the study, revised the work critically for important intellectual content, and approved the final version to be submitted. Ms. Valéria Ferreira Pereira Souza collected the data on motor function, revised the work critically for important intellectual content, and approved the final version to be submitted. Gláucio Andrade Amaral and Marcella Costa Ribeiro Borges collected the data on neuroimaging findings, revised the work critically for important intellectual content, and approved the final version to be submitted. Ms. Lillian Nunes Gomes and Professor Ricardo Khouri collected the data on the laboratory confirmation of Zika virus infection, revised the work critically for important intellectual content, and approved the final version to be submitted. Dr. Patricia da Silva Sousa collected the data on clinical diagnosis and neurological symptoms of the sample, contributed to analysis and interpretation of data, revised the work critically for important intellectual content, and approved the final version to be submitted. Professor Antonio Augusto Moura da Silva is the main researcher of the project "Congenital Zika syndrome," conceived and designed the study, coordinated and supervised data collection, contributed to analysis and interpretation of data, revised the work critically for important intellectual content, and approved the final version to be submitted. All authors approved the final manuscript as submitted and agreed to be accountable for all aspects of the work.

\section{Conflict of Interest}

Dr. Souza reports grants from Brazilian National Council for Scientific and Technological Development (CNPq), State Funding Agency of Maranhao (FAPEMA), Ministry of Health Department of Science and Technology (DECIT), and the Ministry of Education Coordination for the Improvement of Higher Education (CAPES), during the conduct of the study.

Dr. Simoes reports grants from Brazilian National Council for Scientific and Technological Development (CNPq), State Funding Agency of Maranhao (FAPEMA), Ministry of Health Department of Science and Technology (DECIT), and the Ministry of Education Coordination for the Improvement of Higher Education (CAPES), during the conduct of the study.

Dr. Gomes reports grants from Brazilian National Council for Scientific and Technological Development (CNPq), State Funding Agency of Maranhao (FAPEMA), Ministry of Health Department of Science and Technology (DECIT), and the Ministry of Education Coordination for the 
Improvement of Higher Education (CAPES), during the conduct of the study.

Dr. Ribeiro reports grants from Brazilian National Council for Scientific and Technological Development (CNPq), State Funding Agency of Maranhao (FAPEMA), Ministry of Health Department of Science and Technology (DECIT), and the Ministry of Education Coordination for the Improvement of Higher Education (CAPES), during the conduct of the study.

Dr. Amaral reports grants from Brazilian National Council for Scientific and Technological Development (CNPq), State Funding Agency of Maranhao (FAPEMA), Ministry of Health Department of Science and Technology (DECIT), and the Ministry of Education Coordination for the Improvement of Higher Education (CAPES), during the conduct of the study.

Dr. Sousa reports grants from Brazilian National Council for Scientific and Technological Development (CNPq), State Funding Agency of Maranhao (FAPEMA), Ministry of Health Department of Science and Technology (DECIT), and the Ministry of Education Coordination for the Improvement of Higher Education (CAPES), during the conduct of the study.

Dr. Takahasi reports grants from Brazilian National Council for Scientific and Technological Development (CNPq), State Funding Agency of Maranhao (FAPEMA), Ministry of Health Department of Science and Technology (DECIT), and the Ministry of Education Coordination for the Improvement of Higher Education (CAPES), during the conduct of the study.

Dr. Borges reports grants from Brazilian National Council for Scientific and Technological Development (CNPq), State Funding Agency of Maranhao (FAPEMA), Ministry of Health Department of Science and Technology (DECIT), and the Ministry of Education Coordination for the Improvement of Higher Education (CAPES), during the conduct of the study.

Dr. Alves reports grants from Brazilian National Council for Scientific and Technological Development (CNPq), State Funding Agency of Maranhao (FAPEMA), Ministry of Health Department of Science and Technology (DECIT), and the Ministry of Education Coordination for the Improvement of Higher Education (CAPES), during the conduct of the study.

Dr. Khouri reports grants from Brazilian National Council for Scientific and Technological Development (CNPq), State Funding Agency of Maranhao (FAPEMA), Ministry of Health Department of Science and Technology (DECIT), and the Ministry of Education Coordination for the Improvement of Higher Education (CAPES), during the conduct of the study.

Dr. Silva reports grants from Brazilian National Council for Scientific and Technological Development (CNPq), State Funding Agency of Maranhao (FAPEMA), Ministry of Health Department of Science and Technology (DECIT), and the Ministry of Education Coordination for the Improvement of Higher Education (CAPES), during the conduct of the study.

\section{Acknowledgments}

The authors are grateful for the Brazilian National Council for Scientific and Technological Development (CNPq; grant number 440573/2016-5), the State Funding Agency of Maranhao (FAPEMA PPSUS grant number 008/2016), the Ministry of Health Department of Science and Technology (DECIT), and the Ministry of Education Coordination for the Improvement of Higher Education (CAPES; grant number 88881.130813/2016-01) for their financial support. The authors also would like to thank the children and their mothers and/or guardians for having participated in this study.

\section{References}

1 Brito C. Zika virus: a new chapter in the history of medicine. Acta Med Port 2015;28(06):679-680

2 Rasmussen SA, Jamieson DJ, Honein MA, Petersen LR. Zika virus and birth defects-reviewing the evidence for causality. N Engl J Med 2016;374(20):1981-1987

3 WHO WHO statement on the first meeting of the International Health Regulations (2005) (IHR 2005) Emergency Committee on Zika virus and observed increase in neurological disorders and neonatal malformations Available at: http://www.who.int/mediacentre/news/statements/2016/1st-emergency-committee-zika/en/. Accessed September 16, 2020

4 Del Campo M, Feitosa IML, Ribeiro EMZika Embryopathy Task Force-Brazilian Society of Medical Genetics ZETF-SBGM. , et al; . The phenotypic spectrum of congenital Zika syndrome. Am J Med Genet A 2017;173(04):841-857

5 Abuali M, Domachowske J. Congenital and perinatal infections. In: Domachowske J, ed. Introduction to Clinical Infectious Diseases. Springer, Cham; 2019:213-224

6 Collins MH, Waggoner JJ. Detecting vertical Zika transmission: emerging diagnostic approaches for an emerged flavivirus. ACS Infect Dis 2019;5(07):1055-1069

7 Ximenes RAA, Miranda-Filho DB, Brickley EBMicrocephaly Epidemic Research Group (MERG). , et al; . Zika virus infection in pregnancy: establishing a case definition for clinical research on pregnant women with rash in an active transmission setting. PLoS Negl Trop Dis 2019;13(10):e0007763

8 Carvalho A, Brites C, Mochida G, et al. Clinical and neurodevelopmental features in children with cerebral palsy and probable congenital Zika. Brain Dev 2019;41(07):587-594

9 Melo A, Gama GL, Da Silva Júnior RA, et al. Motor function in children with congenital Zika syndrome. Dev Med Child Neurol 2020;62(02):221-226

10 Moura da Silva AA, Ganz JSS, Sousa PD, et al. Early growth and neurologic outcomes of infants with probable congenital Zika virus syndrome. Emerg Infect Dis 2016;22(11):1953-1956

11 Ventura PA, Lage MLC, de Carvalho AL, Fernandes AS, Taguchi TB, Nascimento-Carvalho CM. Early gross motor development among Brazilian children with microcephaly born right after Zika virus infection outbreak. J Dev Behav Pediatr 2020;41(02):134-140

12 Alves LV, Paredes CE, Silva GC, Mello JG, Alves JG. Neurodevelopment of 24 children born in Brazil with congenital Zika syndrome in 2015: a case series study. BMJ Open 2018;8(07):e021304

13 Nielsen-Saines K, Brasil P, Kerin T, et al. Delayed childhood neurodevelopment and neurosensory alterations in the second year of life in a prospective cohort of ZIKV-exposed children. Nat Med 2019;25(08):1213-1217

14 França TLB, Medeiros WR, Souza NL, et al. Growth and development of children with microcephaly associated with congenital zika virus syndrome in Brazil. Int J Environ Res Public Health 2018;15(09):E1990 
15 Marques FJP, Teixeira MCS, Barra RR, et al. Children born with congenital Zika syndrome display atypical gross motor development and a higher risk for cerebral palsy. J Child Neurol 2019;34 (02):81-85

16 Pessoa A, van der Linden V, Yeargin-Allsopp M, et al. Motor abnormalities and epilepsy in infants and children with evidence of congenital Zika virus infection. Pediatrics 2018;141(Suppl 2): S167-S179

17 Moore CA, Staples JE, Dobyns WB, et al. Characterizing the pattern of anomalies in congenital Zika syndrome for pediatric clinicians. JAMA Pediatr 2017;171(03):288-295

18 Russell DJ, Rosenbaum PL, Cadman DT, Gowland C, Hardy S, Jarvis S. The Gross Motor Function Measure: a means to evaluate the effects of physical therapy. Dev Med Child Neurol 1989;31(03): 341-352

19 Frota LMDCP, Sampaio RF, Miranda JL, et al. Children with congenital Zika syndrome: symptoms, comorbidities and gross motor development at 24 months of age. Heliyon 2020;6(06): e04130

20 Massetti T, Herrero D, Alencar J, et al. Clinical characteristics of children with congenital Zika syndrome: a case series. Arq Neuropsiquiatr 2020;78(07):403-411

21 Baer A, Kehn-Hall K. Viral concentration determination through plaque assays: using traditional and novel overlay systems. J Vis Exp 2014;(93):e52065

22 Brasil Ministério da Saúde Secretaria de Vigilância em Saúde Secretaria de Atenção à Saúde Orientações Integradas de Vigilância e Atenção à Saúde No Âmbito Da Emergência de Saúde Pública de Importância Nacional: Procedimentos Para o Monitoramento Das Alterações No Crescimento e Desenvolvimento a Partir Da Gestação Até a Primeira Infância, Relac.. 2017. Available at: http://portalarquivos.saude.gov.br/images/pdf/2016/dezembro/12/orientacoes-integradas-vigilancia-atencao.pdf. Accessed September 16, 2020

23 World Health Organization Screening, assessment and management of neonates and infants with complications associated with Zika virus exposure in utero: Rapid Advice Guideline World Heal Organ. 2016 (WHO/ZIKV/MOC/16.3/Rev3):15. Available at: http://apps.who.int/iris/bitstream/10665/204475/1/WHO_ZIKV_MOC_16.3_eng.pdf?ua=1. Accessed September 16, 2020

24 Palisano R, Rosenbaum P, Walter S, Russell D, Wood E, Galuppi B. Development and reliability of a system to classify gross motor function in children with cerebral palsy. Dev Med Child Neurol 1997;39(04):214-223

25 Hiratuka E, Matsukura TS, Pfeifer LI. Cross-cultural adaptation of the gross motor function classification system into BrazilianPortuguese (GMFCS). Rev Bras Fisioter 2010;14(06):537-544
26 Russell DJ, Rosenbaum PL, Wright M, Avery LMMedida Da Função Motora Grossa [Livro Eletrônico]: [GMFM-88 \& GMFM-66]: Manual Do Usuário. 2a edição. São Paulo: Memnon; 2015

27 Hanna SE, Bartlett DJ, Rivard LM, Russell DJ. Reference curves for the gross motor function measure: percentiles for clinical description and tracking over time among children with cerebral palsy. Phys Ther 2008;88(05):596-607

28 Associação Brasileira de Empresas Pesquisa - ABEP. Critério de Classificação Econômica Brasil [Internet]. São Paulo: Associação Brasileira de Empresas Pesquisa - ABEP; 2015. Available at: http://www.abep.org/criterio-brasil. Accessed May 1, 2020

29 Villar J, Cheikh Ismail L, Victora CGInternational Fetal and Newborn Growth Consortium for the 21st Century (INTERGROWTH21st). , et al; . International standards for newborn weight, length, and head circumference by gestational age and sex: the Newborn Cross-Sectional Study of the INTERGROWTH-21st Project. Lancet 2014;384(9946):857-868

30 The Definition and Classification of Cerebral Palsy Contents Foreword Historical Perspective Definition and Classification Document. The definition and classification of cerebral palsy. Dev Med Child Neurol 2007;49(s109)1-44

31 Rosenbaum PL, Walter SD, Hanna SE, et al. Prognosis for gross motor function in cerebral palsy: creation of motor development curves. JAMA 2002;288(11):1357-1363

32 Zare Mehrjardi M, Poretti A, Huisman TAGM, Werner H, Keshavarz E, Araújo JúniorE. Neuroimaging findings of congenital Zika virus infection: a pictorial essay. Jpn J Radiol 2017;35(03):89-94

33 Marques VM, Santos CS, Santiago IG, et al. Neurological complications of congenital Zika virus infection. Pediatr Neurol 2019; 91:3-10

34 De Góes Cavalcanti LP, Tauil PL, Alencar CH, Oliveira W, Teixeira MM, Heukelbach J. Zika virus infection, associated microcephaly, and low yellow fever vaccination coverage in Brazil: is there any causal link? J Infect Dev Ctries 2016;10(06):563-566

35 Nogueira FC, Velasquez E, Melo AS, Domont GB. Zika virus may not be alone: proteomics associates a bovine-like viral diarrhea virus to microcephaly. Bio Rxiv 2016;16:062596

36 Ferreira HNC, Schiariti V, Regalado ICR, et al. Functioning and disability profile of children with microcephaly associated with congenital Zika virus infection. Int J Environ Res Public Health 2018;15(06):E1107

37 Brasil. Ministério da Saúde. Secretaria de Vigilância em Saúde. Departamento de Vigilância das Doenças Transmissíveis. Protocolo de vigilância e resposta à ocorrência de microcefalia relacionada à infecção pelo vírus Zika / Ministério da Saúde, Secretaria de Vigilância em Saúde, Departamento de Vigilância das Doenças Transmissíveis. - Brasília: Ministério da Saúde, 2015 